\title{
FAST CHEMICAL REACTIONS IN SOLUTION
}

$\mathrm{V}$ ERY many chemical reactions in solution proceed too rapidly to be studied by conventional techniques, which require times of at least several seconds for their manipulation. Slower reactions often involve several steps, all but one of which are fast. It is also generally true that the simplest and most fundamental reactions tend to be fast ones.

The development of electronic methods of detecting and recording has made it possible to record almost any physical parameter in a very short interval of time and therefore to carry out a chemical investigation. But, in order that such an observation shall be significant, it is necessary to initiate the chemical change in some way in a time which is short compared with the time of reaction. Methods of attaining this and some of the results obtained were the subject of a recent meeting organized by the Bunsengesellschaft für physikalische Chemie and the Max-Planck-Gesellschaft. The colloquium was held in Hahnenklee/Harz during September 14-17 and was attended by about one hundred and sixty scientists, of whom about ninety were from Germany, thirty from the United States, twelve from Britain and twenty-eight from other countries. Thirty papers were presented, covering practically every available method.

The first two days were devoted to a discussion of the fast-reaction methods and measuring techniques themselves. Historically the first method was the rapid-mixing method introduced by Hartridge and Roughton thirty-six years ago, and this was discussed by F. J. W. Roughton (University of Cambridge) with particular reference to the reactions of hæmoglobin, for which the method was first developed. It has been extended and modified in many ways, for example, by the introduction of stopped-flow techniques, and is useful for halfreaction times of about 1 millisecond or more. Flash photolysis, developed by Norrish and Porter, is a second generally useful method, and in describing this method G. Porter (University of Sheffield) pointed out that almost every type of reaction can be initiated photochemically, and that at present it is possible to measure bimolecular reaction-rates with velocity constants up to $10^{10}$ litres/mole/sec. and first-order constants up to $10^{5} \mathrm{sec}^{-1}$.

Q. H. Gibson (University of Sheffield) described how the stopped-flow method has been used to study the combination of hæmatin and carboxy-hæm with globin. Examples of the application of the flash photolysis method were given by $\mathrm{H}$. Linschitz and J. Eloranta (Brandeis University), who used this method to study metal and hydrocarbon ion solutions, by H. T. Witt, who gave an extensive survey of the work which he and his co-workers have carried out on chlorophyll with particular reference to primary processes of photosynthesis, and also by $\mathrm{E}$. W. Abrahamson (Syracuse University), who described work on the visual pigments.

Both mixing and flash-photolysis methods are applicable to systems which are far removed from equilibrium as well as to relaxation systems. Most of the other fast-reaction methods start with a system at equilibrium, displace the equilibrium slightly by changing some physical parameter and then observe the rate of relaxation to the new equilibrium position. Dr. M. Eigen and his colleagues at the Max Planck Institute for Physical Chemistry in Göttingen have been pioneers in this field, and a comprehensive review of relaxation methods and an account of the mathematical procedure and of the assumptions necessary to evaluate rate constants from the experimentally determined parameters was given by L. De Maeyer. Displacement can be either periodic or of the jump type, and temperature, pressure, field impulse and sound absorption are all used to produce the disturbance. K. Tamm (University of Göttingen) gave a survey of the use of the sonic relaxation method for rate measurements in water solutions. M. Eigen summarized many reactions which have been studied by relaxation methods/ The fastest reactions which are controlled by dif. fusion give rate-constants of about $10^{10}$ litres/molesec. for ionic reactions other than protonic where values of about $10^{11}$ litres $/ \mathrm{mole} / \mathrm{sec}$. are obtained. The use of the ultrasonic technique to study association reactions and rotational isomerizations was outlined by Van W. Maier (University of Freiburg) and J. Lamb (University of London) respectively, and the application of the relaxation method to enzymatic reactions was discussed by R. A. Alberty and G. G. Hammes (University of Wisconsin).

A new and very powerful approach to fast reactions is provided by the methods of electron and nuclear magnetic resonance. A molecule, such as water or hydrogen peroxide with two equivalent protons, gives a single peak in its nuclear magnetic resonance spectrum. A mixture of water and hydrogen peroxide will give two separate peaks if there is no exchange between the protons of the two molecules. However, if there is a fast exchange of the protons, there will be only one peak as all the protons will then appear equivalent. For intermediate cases the exchange-rate constants may be calculated from the form of the spectra. The nuclear magnetic resonance method can be used for reaction times from 1 to $10^{-3}$ sec. Electron spin resonance spectra can be interpreted in an analogous way to determine reaction-rates with characteristic times in the range $10^{-4}$ to $10^{-9}$ sec.

The carbon dioxide - water equilibrium has been studied by A. Patterson and R. Ettinger (Yale University) using nuclear magnetic resonance methods. Patterson pointed out that two most interesting questions remain unanswered, namely, why is such a large proportion of carbon dioxide in solution present in dissolved but not hydrated form, and, secondly, why is the hydration reaction such a slow process. R. G. Pearson (Northwestern University) explained how paramagnetic ions in solution affect the line-shape and how line-width can be used to measure exchange rate constants for reactions of complex ions.

Methods which are not quite so general in their application include the fluorescenee method-A. Weller (Technischer Hochschule, Stuttgart) described how it is used to measure the fast reactions of excited molecules-and electrochemical methods. R. Brdicka (University of Prague), Jiri Koryta (Academy of 
Science, Prague) and others gave comprehensive reviews on the use of polarographic technique in the study of electrode reactions.

The transfer of electronic excitation energy between molecules is a special type of fast reaction which, in some cases, is so efficient that rate constants exceed those calculated for the maximum rate of a diffusion-controlled reaction using normal collision diameters. Prof. Th. Förster (Technischer Hochschule, Stuttgart) explained how the interaction in such cases occurs through dipole-dipole coupling so that energy transfer can take place over distances as great as $50 \mathrm{~A}$. Two examples of the direct measurement of energy transfer processes were presented. S. S. Brody (University of Illinois) has used a small specially designed hydrogen lamp to produce a flash of a few millimicroseconds duration. He measured the delay between the flash and the fluorescence of chlorophyll in the chloroplast. By using suitable filters, other pigments apart from chlorophyll were made to absorb the light and the sensitized fluorescence of chlorophyll was studied. M. Burton and H. Dreeskamp (University of Notre Dame) were also able to resolve decay times of about $10^{-9} \mathrm{sec}$. A Iow-voltage X-ray tube was used to deliver an approximately square wave pulse of about $10^{-8}$ sec. duration with rise and fall times each about $10^{-9}$ sec. By this means the energy transfer from benzene to $p$-terphenyl was measured.

Papers by H. Eyring (University of Utah) and R. M. Noyes (University of Oregon) dealt with questions of solution structure and fast reactions.
Noyes has developed a simple theory to predict the quantum yield for iodine dissociation from the values of the viscosity of the solvent, the sizes and masses of the fragments of dissociation, the energy of the bond that is broken and the energy of the absorbed quantum. The agreement between theory and experiment is remarkably good for such a simple model.

A very pleasant feature of the meeting was a visit to the Max Planck Institute in Göttingen, where a number of experiments on fast reactions in solution were demonstrated. This visit provided a unique opportunity to compare and contrast many of the methods discussed at the colloquium. The methods demonstrated included (1) flow methods, (2) relaxation methods-field impulse, temperature and pressure jump and sound absorption, (3) polarographic methods, and (4) the nuclear magnetic resonance method.

A lively discussion followed most of the sessions and was greatly facilitated by an excellent earphone translation system between the German and English languages. Most of us left the meeting feeling that, with such a wealth of excellent methods available, there is now practically no chemical reaction in which rate cannot be directly measured, and the 'instan. taneous reaction' has gone for ever. It was also clear that reactions which proceed in times of less than one second are as numerous and at least as funda. mentally important as those which allow a more leisurely approach to rate measurement.
G. Porter
F. WILKINSON

\section{SWISS SOCIETY FOR NATURAL SCIENCES}

$\mathrm{T}$ HE one hundred and thirty-ninth annual meeting of the Swiss Society for Natural Sciences was held at Lausanne during September 11-13. The annual president, Prof. A. Girardet (Lausanne), opened it, with an address on "La science, le savant et le pharmacien", touching the history and psychology of pharmaceutical teaching, practice and research, which in Switzerland are the concern of the universities and medical authorities far more closely than in Britain in the general pattern of scientific and industrial development. He pointed out in conclusion the financial handicap implied by the present-day loss of monopoly for the sale of many types of drugs, which has forced the pharmacist to extend his business to non-pharmaceutical products to the detriment of his professional standing. Once the equals of the surgeon in the employ of the medical theorist, the pharmacist and pharmacologist have not fought their way to the same independence and supremacy as he has.

The first general meeting adjourned to St. Croix in the Jura Mountains, a geological and industrial district of considerable interest, in magnificent weather enjoyed with mixed feelings by the inhabitants, entirely dependent on wells for their water. Prof. A. de Muralt (Berne) reported on the first seven years of the Swiss National Fund for Scientific Research, State subsidized but remarkably free from State control, and available to Swiss organizations and individuals in the widest conceivable manner. Nuclear research has naturally come in for a large share, and its potentialities and limitations in Switzer- land were discussed separately in more detail by Prof. P. Hüber (Basle), who has been most concerned with it.

The remainder of the general proceedings took place in the fine painted Aula of the University of Lausanne, adjoining the magnificently re-organized up-to-date library and the Museum of Fine Arts, where one of the hospitable receptions was held, all of them pleasantly devoid of any visible host or formality of any kind.

After an illuminating account by $P$. Muller, of the Paris Observatory, on "Le Pourquoi des Satellites Artificiels", and a masterly and moving survey, "La Science à la Recherche d'une Conscience", by the annual vice-president, Prof. D. Rivier (Lausanne), the day ended with a report by DT. G. Chevallaz (syndic of Lausanne) on preparations in hand for the Swiss National Exhibition to be held in 1964 at Lausanne : in better circumstances, it is to be hoped, than its predecessors of 1914 in Berne and 1939 in Zurich.

Finally, at the third general meeting, Dr. F. Kaudewitz, of the Max Planck Institute of 'Tübingen, spoke on "Methoden, Denkweise und Ergebnisse der Erbforschung an Bakterien", with a sober simplicity, clarity and directness remarkable in so new and complex a domain; and the sous-directeur of the National Museum of Natural History at Paris, T. Dorst, under the title "La Signification des Galapagos pour l'Étude de l'Évolution', gave the latest findings and hypotheses on these unique islands. 